\title{
"DISPOSICIONES PSICOLÓGICAS ANTE LOS DIFERENTES TIPOS DE AFRONTE A ESTADOS DE EMERGENCIA DE ORIGEN NATURAL Y SOCIAL"
}

\author{
Luis Alberto Vicuña Peri \\ Ps.Héctor Hernandez Valz \\ Mg. Mildred Paredes Tarazona \\ Ps.Rolando Solís Narro \\ Ps.Jonan Pecho Gallarza
}

\begin{abstract}
RESUMEN
La presente investigación comparó en función a la edad cronológica, estado civil, género y tipo de ocupación las Disposiciones Psicológicas ante los estados de emergencia de origen natural y social de 776 sujetos de Lima, cuyo tamaño de error muestral fue del 0.05, con un nivel de confianza del 0.95. La información acerca de las disposiciones psicológicas se obtuvo mediante una escala ordinal. Los datos se analizaron con el análisis de varianza para datos ordinales de una, dos y tres vías según los cruces de las variables. Las variables arriba señaladas no influyen sobre las Disposiciones Psicológicas y se puede afirmar que las reacciones de las personas son comunes en cuanto a la conservación tanto de la vida como de la integridad física. Los desastres de origen natural tales como terremotos, tsunamis e incendios son los que generan más temor en la población; el lugar preferido en caso de ocurrencia es el hogar, o volver a él a cualquier costo. Consideran a Defensa Civil, a la Cruz Roja y a los Bomberos como efectivos en la ayuda, pero esto no llega en su totalidad a los afectados y que los datos se reducen mediante la prevención.

Con respecto a los desastres de origen social tales como el terrorismo, las pandillas juveniles y los asaltos a mano armada, producen mucho temor, y la reacción ante ellos es correr hacia un lugar seguro, dar la voz de alarma y hacer la denuncia respectiva.
\end{abstract}

PALABRAS CLAVE: Convergencia, Foco de desastre, desastre social, desastre natural, disposiciones psicológicas.

\begin{abstract}
The present research compared on the basis of chronological age, civil state, gender and type of business the psychological aptitudes facing the emergency states with natural and social origin in 776 persons in Lima, whose sample of mistake size was 0,05, with a confidence level of 0,95. The informations about psychological aptitudes obtained through an ordinal scale. The data analyzed with the varianza's analysis to ordinal data of one, two and three processes according to the intersections of variables. The variables above indicated not influence over psychological aptitudes and we can assert that people reactions are common as for the preservations as about life as physical integrity. The natural disasters such as earthquakes, tsunamis and fires generate more fear in population; the favorite place where they happen is the home. they consider a Civil Defence, The Red Cross and The Fire Brigade like help, but this doesn't reach to the affected, and damages reduce throught prevention.

With respect to social damages such as Terrorism, Young Gangs and Assaults, they cause a lot of fear; and the reaction in front of those it is run towards an safe place, to alert and make the denounce.
\end{abstract}

KEY WORDS: Convergence, damage focus, social damage, natural damage, psychological aptitudes. 
Recién a partir de 1950 comenzaron los estudios científicos de los desastres y otras situaciones de emergencia, anteriormente la mayor parte de la literatura sobre el comportamiento colectivo en estados de emergencia estaba constituida por descripciones periodísticas de las que se desprendieron los modelos teóricos, analizaban el comportamiento colectivo en términos del estado individual y su irracionalidad como en los trabajos de Le Bonn. En cambio los estudios científicos de tales emergencias señalaron la naturaleza fuera de lo común de las mismas, como una desviación de la rutina y normas establecidas en la vida diaria, y proporcionales a lo extenso e inesperado de la emergencia. La situación de emergencia, así como su naturaleza colectiva produce efectos diferenciales sobre el comportamiento; sin embargo muchos ejemplos de pánico, huida y heroísmo se convierten en metas directas y comprensibles. Por ejemplo Turner y Killan, 1957 encontraron que comportamientos irracionales durante y después de explosiones o huracanes, constituían esfuerzos notorios de los individuos por llegar hasta donde estaban sus familiares, salvar a sus niños, o en unos cuantos casos, cumplir con su misión comunitaria como policías, bomberos etc.

Los componentes de una emergencia, cualquiera que sea su naturaleza específica, no son solo eventos sensoriales inusitados con características impresiones e impactantes. Son señales de peligro o de hechos catastróficos reales que requieren acción rápida o inmediata solución para poder sobrevivir, las emergencias llegan a ser el punto focal dentro de la situación en la cual ocurren, siendo indicativas de peligro real o percibido y de posible catástrofe. Por eso el foco de una emergencia lo constituye una situación urgente y problemática que debe ser atendida y solucionada sin demora. Ante el foco de una emergencia la persona involucrada hará lo apropiado para sobrevivir, superada la crisis surge el comportamiento convergente que puede ser: pasivo, ansioso, solidario, curioso y explotadores, comportamiento que puede obstaculizar las operaciones de auxilio por lo que se amerita su estudio.

El Perú por su ubicación geográfica dentro del llamado círculo de fuego, más la cordillera de los andes y la corriente fría de Humboldt nos hace un país expuesto a condiciones naturales que en cualquier momento puede generar un foco de emergencia, sea por movimientos telúricos, huaycos y el fenómeno del niño con sus manifestaciones en forma de lluvias torrenciales en algunas regiones del país y sequías en otras de sus regiones. A estas condiciones naturales se les suma las condiciones sociales tales como jerarquizaciones de valores reñidas con lo que debería esperarse para satisfacer nuestras necesidades de salud, vivienda, educación, diversión y saneamiento. Estas jerarquizaciones han generado un crecimiento urbano al margen de planes de desarrollo que no tienen como eje principal la prevención de desastres y evitar el establecimiento de comunidades en zonas de alto riesgo, haciéndolas vulnerables no solo a los desastres de causa natural sino también a los de origen social que a la fecha se exteriorizan en las pandillas juveniles, en la violencia familiar y en la búsqueda de cultos que consuele y restaure la esperanza aun cuando para ello expongan no solo la mejor calidad de vida 
sino hasta la suya propia, como el caso de los clubes de "fans".

Esta descripción deja entrever que no hemos aprendido de la historia, que no hemos considerado que el hombre precolombino supo interactuar con los fenómenos naturales, como expresa el antropólogo Ruben Wong, docente investigador de la Universidad Nacional Mayor de San Marcos, quien afirma que éstos localizaron sus viviendas en las partes altas y nunca en los lechos de los ríos secos, como se viene haciendo en la actualidad que la urbe está en contradicción a las leyes de la naturaleza. Por otro lado el Coronel de la Policía Nacional del Perú, José Villanueva Garay, experto en defensa Civil, sostiene que una característica muy difundida del habitante peruano es relacionar las situaciones de amenaza o de riesgo potencial con creencias religiosas como la que ocurrió en Lima a raíz del gran terremoto a las 22 horas del 28 de Octubre de 1746, seguida luego por un tsunami que llegó hasta Carmen de la Legua, el habitante no tomó medidas preventivas sino se protegió en un nuevo culto religioso que hasta la fecha se venera, (el Señor de los Milagros o el mes morado) no afirmamos que esta reacción esté mal, es lo contrario ayuda al hombre a recuperar su estado de equilibrio emocional, lo malo está si solo se queda en el ritualismo y no se asumen las medidas materiales de prevención. Así mismo nos dice el mencionado Coronel que Defensa Civil hace 26 años que ha iniciado campañas de difusión orientadas a sensibilizar a la población ante estados de emergencia, pero no han logrado cambios significativos, en gran medida agrega porque los aspectos legales no son lo suficientemente efectivos en lo que respecta al desarrollo de la urbe, se autorizan y se conceden terrenos para vivienda sin previo estudio de su exposición a la amenaza o riesgo potencial natural a tal extremo que si ocurriera un sismo de alta intensidad la esperanza matemática de muertes esta alrededor del 15\%. Por otro lado el psicólogo Santiago Valero, dedicado a la Compañía de Bomberos Voluntarios del Perú, ha efectuado estudios observacionales y bibliográficos acerca del impacto psicológico que generan los desastres enfatizando en el gran sentimiento de sufrimiento que padecen las personas que en breves minutos lo han perdido todo y la secuela emocional asociado a prolongados estados de estrés. Como medida de ayuda desde 1986 ha organizado el primer grupo de psicólogos capacitados en simulacros de sismo en Provincia Constitucional del Callao, a la fecha continua con sus estudios buscando optimizar la asistencia del apoyo psicológico a las victimas de un desastre. Por su parte Calderón, C. De la Cruz Roja comunica que en su institución vienen desarrollando programas para capacitar en apoyo psicológico orientado a las zonas de desastre, comunica que una de sus principales dificultades radica en los prejuicios y creencias que poseen las víctimas del desastre, y por otro lado ponen especial atención en el estudio de la dinámica de los fenómenos naturales con el propósito de aumentar la eficacia de los programas de anticipación ante lo inevitable. 
Ante estos hecho y condiciones reales resulta de primer orden explorar las disposiciones psicológicas, por ahora del habitante limeño, ante los focos de emergencia, entendemos que las diferentes organizaciones arriba mencionadas trabajan con ahinco, pero chocan con la realidad cuando las víctimas potenciales no asumen compromiso para aceptar y tomar en serio por ejemplo los simulacros de focos de emergencia y tampoco otras recomendaciones que, al margen del nivel económico, de practicarse evitarían perdidas que en muchos casos son irrecuperables, a este componente psicológico lo denominamos disposición, y se refiere a la prevalencia que los sujetos otorgan a sus significados ante estados de emergencia. Los significados son la resultante de experiencias directas o indirectas, manifiestas o latentes que los sujetos formamos en torno a una persona, objeto, hecho o fenómeno con componente cognitivos y emocionales. El componente emocional es el que comúnmente prima, de allí que las personas suelen buscar repuestas incoherentes con la realidad apelando a respuestas en lo mágico, fantasioso o divino, en otros casos se combinan lo emocional con la oportunidad que la situación plantea para obtener un beneficio personal y, otras veces, nuevamente con predominio emocional sentirse importante por haber sido testigo presencial de un foco de emergencia.

Estos significados pueden variar en función al género, la edad, el tipo de ocupación y el estado civil. El género debido a la diferencia que se encuentra entre varones y mujeres respecto al componente emocional; sin embargo la edad por el proceso madurativo que implícitamente lleva puede actuar atenuándola. El tipo de ocupación puede ser otra variable diferenciadora pata tal significado ya que no es lo mismo el papel de dependiente respecto a quienes tienen permanentemente que decir incluyendo la inversión económica y el riesgo de pérdida. Por otro lado el estado civil promueve matices emocionales muy variados que van desde la reacción depresiva extrema hasta actos de verdadero heroísmo.

De lo descrito, cuando trabajamos con una muestra de limeños varones y mujeres de 15 años hacia adelante, con diferentes tipos de ocupación (para efectos del estudio solo se consideran las siguientes categorías: estudiante, empleado, obrero y comerciante), estado civil (solteros, casados, separados o divorciados) queremos despejar la siguiente interrogante: ¿El género, la edad, el tipo de ocupación y el estado civil influyen sobre las disposiciones psicológicas ante los focos de emergencia de origen natural y social?, estas variables ¿son independientes o interactuantes sobre las disposiciones psicológicas ante los focos de emergencia de origen natural y social? 


\section{DEFINICIÓN DE TÉRMINOS}

\section{Focos de emergencia de origen natural:}

Son fenómenos que ocurren sorpresivamente, de manera explosiva provocando daños personales y materiales de magnitudes diferentes, ejemplos de ello son terremotos, temblores de tierra, aluviones. inundaciones, incendios, tormentas, tsunamis, vientos huracanados, sequias.

\section{Focos de emergencia de origen social:}

Son fenómenos que ocurren luego de una acción planificada con sin plataforma de reinvindicaciones, donde los participantes comparten los agravios que les llegan de un sector de la comunidad cuyas acciones son capaces de provocar daños en las personas y en las propiedades públicas y privadas así también a los daños por falta de previsión industrial y urbana se presentan como: acciones individuales y colectivos capaces de causar daño a personas y bienes tales como: marchas de protesta, asociadas a la destrucción o autodestrucción,enfrentamiento entre pandillas, tumulto improvisado espectáculos públicos, la contaminacion medio-ambiental, usurpaciones.

\section{Disposiciones psicológicas:}

Proceso psicológico de redes de significados que predispone al individuo para pensar, sentir y actuar de una forma determinada entre varias posibles que en la presente investigación se manifiestan como priorizaciones de conductas ante los focos de emergencia de origen natural y social.

\section{Estudiante:}

Persona cuya actividad prioritaria es la actividad académica regular sea de nivel secundario como de nivel superior.

\section{Empleado:}

Caracterizado por relación contractual permanente o temporal con el empleador cuyo trabajo es prioritariamente intelectual.

\section{Obrero:}

En condición similar al empleado pero cuyo trabajo es prioritariamente manual.

\section{Comerciante:}

Dueño del negocio cuya actividad es la transacción económica por intercambio de bienes y productos 


\section{HIPÓTESIS}

1. Se presupone que las disposiciones psicológicas ante los focos de emergencia de origen natural y social difieren en función al género, edad cronológica, estado civil y tipo de ocupación.

2. Las variables género, edad cronológica, estado civil y tipo de ocupación interactúan sobre las disposiciones psicológicas ante los focos de emergencia de origen natural y social.

\section{MATERIAL Y MÉTODOS}

El estudio realizado es descriptivo comparativo teniendo como variable dependiente las disposiciones psicológicas ante los focos de emergencia de origen natural y social y como variables independientes el género, edad cronológica, estado civil y tipo de ocupación.

La población de Lima metropolitana según datos del INEI - 1993 para sujetos comprendidos entre los 15 a más edad y distribuidos en cinco conos ( Centro, Norte, Sur, Este y Oeste) es de 3'987,003 habitantes.

La Muestra de trabajo fue probabilística y por conglomerados calculando un tamaño total con un riesgo de error del 0.05 y un nivel de confianza del $95 \%$. cuyo tamaño fue estimado en 739 sujetos, sin embargo para prevenir cuestionarios defectuosamente contestados se fijó en 776 elementos muestrales con afijaciones proporcionales en función al género y conos poblacionales. Quedando la muestra constituida por 386 varones y 390 mujeres. Así mismo 424 fueron solteros, 315 casados y 37 en otros estados civiles (separados, divorciado). En cuanto a la ocupación 247 fueron estudiantes, 210 empleados, 166 obreros y 153 comerciantes.

El instrumento de recolección da datos fue elaborado especialmente para la presente investigación y denominado "Escala de Disposiciones Psicológicas ante los diferentes tipos de afronte a Estados de Emergencia de Origen Natural y Social ", cuyas siglas son D.P.E.E.N.S.-1998. Se trata de una escala de 18 situaciones estructuradas, de dos dimensiones relativas a estados de emergencia, donde la primera dimensión, constituida por 11 situaciones estructuradas, corresponde a focos de emergencia natural, y la segunda dimensión, constituida por 8 situaciones estructuradas, corresponden a focos de emergencia social. Cada situación estructurada plantea ítems para que el examinado conteste jerarquizando su respuesta, lo que caracteriza a la prueba como una escala ordinal. La primera dimensión se refiere a la localización de la persona en el momento de la ocurrencia del desastre, ubicándolo en su hogar, en la calle o en el centro de labores. Los indicadores para la presente dimensión fueron: Pasividad, ansiedad, solidaridad, curiosidad y explotador de la situación. La segunda dimensión se refiere al comportamiento social, organización social, componentes culturales y medios para afrontar, siendo sus indicadores: 
Importancia de la seguridad del otro, compromiso con la prevención, labor conjunta con ganancia individual, curiosidad, pillaje, prensa sensacionalista, aprovechamiento de la ayuda, participación de instituciones como defensa Civil, La Cruz Roja, Los Bomberos, La Fuerzas Armadas y Policiales y el Gobierno así también la memoria social de desastres, creencias, mitos y tabúes y los programas colectivos de prevención.

La validez tanto de las 18 situaciones estructuradas como de los ítemes para cada una de las situaciones estructuradas, fue por el análisis de contenido solicitando la opinión de expertos. Originalmente fueron 20 las situaciones estructuradas quedando después de la validez en el número antes mencionado. Solo se aceptó las situaciones como los itemes si resultaban significativas a la opinión de los jueces a un margen de error no mayor del 0.01 mediante el análisis con el Ji cuadrado. Así mismo se efectuó con un grupo piloto de 50 sujetos con características similares que iban a configurar la muestra el análisis semántico con el propósito de verificar que los vocablos utilizados denoten y connoten lo que los autores se propusieron, el resultado de este análisis fue positivo.

La confiabilidad fue por el método de la doble aplicación en dos momentos diferentes a la misma muestra, luego las respuestas a cada Ítem para cada situación estructurada fueron correlacionadas utilizando la fórmula de correlación por rangos de Spearman encontrando coeficientes que fueron de un mínimo de 0.94 hasta un máximo de 0.98. Demostrándose así que la escala elaborada es confiable.

La recolección de información fue mediante aplicaciones de la escala en forma colectiva para los estudiantes en grupos no mayores de 25 por vez, se encontró en todos los casos motivación y deseo de contestar la escala. Para la muestra de empleados, obreros y comerciantes la aplicación fue de forma individual, contando con la valiosa colaboración de nuestros alumnos de la promoción 1998 de la Facultad de Psicología quienes luego de ser capacitados en las situaciones previas durante y después del examen, se les dio una cuota fija de aplicación con instrucciones precisas según el requerimiento de la muestra, este proceso demoró dos meses cuidando siempre de los controles de veracidad de los datos, al parecer la aplicación individual para estos sectores de la población dio resultados favorables porque no tuvimos que eliminar ningún protocolo de los 776 sujetos muestrales.

Una vez concluida la aplicación se elaboraron las bases de datos para someter al estadístico del análisis de varianza para escalas ordínales e interaccíones con un margen de error no mayor del 0.01 adícionalmente se cálculo las medias aritméticas por cada Ítem para obtener la jerarquización de las respuestas de la muestra examinada.

\section{RESULTADOS}

Los resultados que a continuación presentamos son el producto de haber sometido los datos recolectados al análisis de varianza para cuatro dimensiones con sus respectivas vías: la edad (en tres grupos 15 a 24 años, 25 a 39 años y de 40a a más), la ocupación (estudiante, empleado, obrero y comerciante), estado civil (soltero, casado y en un solo 
grupo separados y divorciados) y el género (varones y mujeres). Este análisis nos permite establecer si hay diferencias endo y exogrupo en las Disposiciones Psicológicas ante los focos de emergencia en función a las variables: edad, género, estado civil y ocupación, y adicionalmente hemos calculado las medias aritméticas para cada una de las dimensiones y vías con el propósito de jerarquizar las respuestas en cada uno de los itemes. Empezaremos por analizar las disposiciones Psicológicas ante estados de emergencia de origen natural para presentar finalmente los resultados ante los focos de emergencia de origen social.

A. Las disposiciones psicológicas ante los focos de emergencia de origen natural en función al género, edad, estado civil y tipo de ocupación.

1. Las reacciones de miedo y los desastres naturales, en la jerarquización que efectúan los examinados entre huayco, incendio, huracanes, tormenta y tsunamis se encontró que las reacciones de miedo antes estos no difieren en función a las variables edad. genero, ocupación y estado civil.

El fenómeno que produce reacciones de mayor miedo en toda la muestra estudiada son los terremotos, luego los incendios y tsunamis, no generan miedo los huaycos, huracanes y tormentas como se puede ver en el Cuadro Nro.1

\section{Cuadro Nro 01}

Resumen de Análisis de varianza (Razones F) en función a la edad, género y estado civil y medias aritméticas de la muestra total (776 sujetos) respecto a los desastres naturales que producen miedo.

\begin{tabular}{|l|c|c|c|c|c|}
\hline $\begin{array}{l}\text { Tipos de } \\
\text { Desastre }\end{array}$ & $\begin{array}{c}\text { Edad } \\
\text { F }\end{array}$ & $\begin{array}{c}\text { Gén. } \\
\text { F }\end{array}$ & $\begin{array}{c}\text { E.C } \\
\text { F }\end{array}$ & $\begin{array}{c}\text { Prom } \\
\text { Gp.T. }\end{array}$ & Rango \\
\hline Huayco & $\mathbf{4 . 5 2}$ & $\mathbf{0 , 0 2}$ & $\mathbf{2 , 4 6}$ & $\mathbf{3 . 8 0}$ & $\mathbf{4}$ \\
Terremoto & $\mathbf{0 . 6 6}$ & $\mathbf{2 0 . 4}$ & $\mathbf{0 . 4 7}$ & $\mathbf{2 . 1 6}$ & $\mathbf{2}$ \\
Incendio & $\mathbf{0 . 0 8}$ & $\mathbf{2 . 7}$ & $\mathbf{1 . 1 4}$ & $\mathbf{3 . 2 4}$ & $\mathbf{3}$ \\
Huracán & $\mathbf{4 . 9 9 *}$ & $\mathbf{1 0 . 5}$ & $\mathbf{1 . 3 4}$ & $\mathbf{3 . 7 7}$ & $\mathbf{4}$ \\
Tormentas & $\mathbf{0 2}$ & $\mathbf{0 . 6 9}$ & $\mathbf{0 . 0 3}$ & $\mathbf{4 . 7 0}$ & $\mathbf{5}$ \\
tsunamis & $\mathbf{0 . 3 2}$ & $\mathbf{2 . 7 9}$ & $\mathbf{0 . 2 2}$ & $\mathbf{3 . 2 9}$ & $\mathbf{3}$ \\
\hline
\end{tabular}

(*)F significativa al 0.01 de margen de error

2. Lugar preferido en caso de ocurrir el desastre que produce mayor temor: La muestra en general elige el hogar como el lugar preferido, habiendo mayor incidencia en el sector femenino, en segundo lugar lo ocupan indistintamente estar en la calle o en el centro de labores. (Ver Cuadro Nro.2) 


\section{Cuadro Nro 02}

Resumen de Análisis de varianza (Razones F) en función a la edad, género y estado civil y medias aritméticas de la muestra total (776 sujetos) respecto al lugar en que quisiera estar de ocurrir un desastre de origen natural.

\begin{tabular}{|l|c|c|c|c|c|}
\hline Lugares & $\begin{array}{c}\text { Edad } \\
\text { F }\end{array}$ & $\begin{array}{c}\text { Gén. } \\
\text { F }\end{array}$ & $\begin{array}{c}\text { E.C } \\
\text { F }\end{array}$ & $\begin{array}{l}\text { Prom } \\
\text { Gp.T. }\end{array}$ & Rango \\
\hline & & & & & \\
En el hogar & 0.48 & 1.91 & 4.89 & 1.32 & 1 \\
En la calle & 2.13 & $16.8 *$ & 131 & 2.3 & 2 \\
En el Centro & 0.16 & 6.46 & 0.05 & 2.4 & 3 \\
de labores & & & & & \\
\hline
\end{tabular}

(*)F sígnificativa al 0.01 de margen de error.

3. Lo que probablemente se haga finalizado el desastre de origen natural: El genero y la edad en forma independiente influyen sobre la disposición psicológica de ponerse a rezar en dirección a las personas de 40 a más edad y a las mujeres. La muestra en general ubica en primer lugar ir a su casa en caso de haber estado fuera, en segundo lugar pedirían ayuda y en tercer lugar irían al foco del desastre, lo que casi no se haría ante lo arriba señalado es primero ponerse a rezar o a pensar en lo que se debe hacer. (Ver Cuadro Nro. 3)

\section{Cuadro Nro 03}

Resumen de Análisis de varianza (Razones F) en función a la edad, género y estado civil y medias aritmeticas de la muestra total (776 sujetos) respecto a lo que se hará una vez que terminó el desastre natural.

\begin{tabular}{|l|c|c|c|c|c|}
\hline Lo que se hará & $\begin{array}{c}\text { Edad } \\
\text { F }\end{array}$ & $\begin{array}{c}\text { Gén. } \\
\text { F }\end{array}$ & $\begin{array}{c}\text { E.C } \\
\text { F }\end{array}$ & $\begin{array}{c}\text { Prom } \\
\text { Gp.T. }\end{array}$ & Rango \\
\hline Regresar a mi hogar & 3.67 & $\mathbf{0 2 3}$ & $\mathbf{0 . 7 5}$ & $\mathbf{1 . 7 6}$ & $\mathbf{1}$ \\
\hline Ir al foco del desastre & $\mathbf{0 . 0 1}$ & $\mathbf{2 . 8 7}$ & $\mathbf{0 . 0 5}$ & $\mathbf{3 . 6 9}$ & $\mathbf{3}$ \\
\hline Pedir ayuda & 2.75 & $\mathbf{0 . 0 7}$ & $\mathbf{0 . 1 0}$ & $\mathbf{2 . 7 6}$ & $\mathbf{2}$ \\
\hline Ponerse a rezar & $\mathbf{1 1 . 6 9 *}$ & $\mathbf{6 . 7 6} *$ & $* \mathbf{0 . 6 8}$ & $\mathbf{3 . 7 5}$ & 4 \\
\hline Pensar en qué hacer & $\mathbf{2 . 4 4}$ & $\mathbf{0 . 1 0}$ & $\mathbf{6 . 9 4}$ & $\mathbf{3 . 7 5}$ & 4 \\
\hline
\end{tabular}

(*) F significativa al 0.01 de margen de error

4. Autopercepción del estado emocional de como se quedará después de un desastre: La edad y el género, en forma independiente. influyen en los sujetos en general de 40 o más años y las mujeres también en general creen que quedarían con mucho temor a que vuelva a repetirse el desastre amargados, traumados resentidos con todo y arrepentidos por no haber tomado precauciones En la muestra general el temor que vuelva a repetirse el desastre ocupa el primer plano, seguido del arrepentimiento por 
no haber tomado precauciones aceptando lo ocurrido, y principalmente los sujetos de 40 a más edad dicen que quedarían traumados por mucho tiempo, el sentimiento que tienen menor probabilidad de ocurrencia masiva es el de sentirse amargado y resentido con todo. (Ver Cuadro Nro. 4)

\section{Cuadro Nro 04}

Resumen de Análisis de varianza (Razones F) en función a la edad, género y estallo civil y medias aritméticas de la muestra total (776 sujetos) respecto a como creen que quedaran después de un desastre natural.

\begin{tabular}{|l|c|c|c|c|c|}
\hline Como se cree que se va a quedar & Edad & Gén. & EC & Prom & Rango \\
\hline Temeroso de replicas & F & F & F & Gp.T. & (1.55 \\
\hline Traumado por tiempo & $\mathbf{1 . 2 0}$ & $\mathbf{2 . 0 3}$ & 1 \\
\hline Amargado con todos & $\mathbf{5 3 0}$ & $\mathbf{9 . 5 9}$ & $\mathbf{0 . 0 1}$ & $\mathbf{3 . 6 9}$ & $\mathbf{4}$ \\
\hline Arrepentido por no haber tomado precauciones & $\mathbf{0 . 5 3}$ & $\mathbf{1 0 . 3 2}$ & $\mathbf{0 . 0 4}$ & $\mathbf{4 . 0 9}$ & $\mathbf{5}$ \\
\hline Acepto lo ocurrido & $\mathbf{0 . 9 2}$ & $\mathbf{7 . 2 4} *$ & $\mathbf{0 . 0 2}$ & $\mathbf{1 . 6 0}$ & $\mathbf{2}$ \\
\hline
\end{tabular}

(*) F significativa al 0.01 de margen de error.

5. Percepción de la efectividad de las instituciones pertinentes: La edad y el estado civil interactúan sobre la Disposición Psicológica a favor de defensa civil en dirección a los solteros con edad igualo menores de 39 años. A su vez la muestra en general considera a Defensa Civil como una institución efectiva ante los desastres, en segundo lugar ubican a la Cruz Roja, en tercer lugar a los Bomberos, en tanto que no se considera efectivo al Gobiemo y menos a las Municipalidades. (Ver Cuadro Nro. 5)

\section{Cuadro Nro. 05}

Resumen de Análisis de varianza (Razones $F$ ) en función a la edad, género y estado civil y medias aritméticas de la muestra total (776 sujetos) respecto a la percepción de la efectividad de las instituciones para enfrentar los desastres de origen natural.

\begin{tabular}{|l|c|c|c|c|c|}
\hline $\begin{array}{l}\text { Percepción efectividad } \\
\text { instituciones }\end{array}$ & $\begin{array}{c}\text { Edad } \\
\text { F }\end{array}$ & $\begin{array}{c}\text { Gen. } \\
\text { F }\end{array}$ & $\begin{array}{c}\text { E.C. } \\
\text { F }\end{array}$ & $\begin{array}{c}\text { Prom. } \\
\text { Gp.T. }\end{array}$ & Rango \\
\hline Def. Civil & 128 & 0.59 & $0.3^{*}$ & 1.82 & 1 \\
\hline Cruz Roja & 0.85 & 2.99 & 2.18 & 2.56 & 2 \\
\hline Bomberos & 3.63 & 0.77 & 0.0 & 2.77 & 3 \\
\hline FFAA y PNP & 1.48 & 1.43 & 6.68 & 4.06 & 4 \\
\hline El Gobierno & 0.59 & 3.36 & 1.58 & 4.66 & 5 \\
\hline Municipios & $\mathbf{0 2 9}$ & $\mathbf{6 . 9 6}$ & $\mathbf{0 . 9 3}$ & $\mathbf{5 . 0 7}$ & $\mathbf{6}$ \\
\hline
\end{tabular}

(*) F significativa 0.10 de margen de error.

6. Repercusiones cuando nos olvidamos de la historia: La edad influye sobre la 
Disposición psicológica de considerar que los desastres son mayores porque se olvida que ya han pasado antes en dirección a los sujetos de 40 o más años. En tanto que los de 24 a menos edad dicen que son peores los desastres porque la gente espera que otros les den seguridad. Y la muestra en general menciona que los desastres son mayores porque se ocupan zonas de alto riesgo, luego porque se posterga la toma de medidas preventivas, también porque nos olvidamos de los comités de defensa y que ya han pasado antes. (Ver Cuadro Nro. 6)

\section{Cuadro Nro 6}

Resumen de Análisis de varianza (Razones F) en función a la edad, género J' estado civil y medias aritméticas de la muestra total (776 sujetos) respecto a la percepción de la magnitud de los desastres de origen natural cuando con el pasar del tiempo

\begin{tabular}{|l|c|c|c|c|c|}
\hline Percepc. & $\begin{array}{c}\text { Edad } \\
\text { F }\end{array}$ & $\begin{array}{c}\text { Gen. } \\
\text { F }\end{array}$ & $\begin{array}{c}\text { E.C } \\
\text { F }\end{array}$ & $\begin{array}{c}\text { Prom } \\
\text { Gp.T }\end{array}$ & Rango \\
\hline $\mathbf{A}^{* *}$ & 2,86 & 3.30 & 0.14 & 2.93 & 3 \\
\hline $\mathbf{B}^{* *}$ & $9.59^{*}$ & 4.45 & 0.48 & 2.93 & 3 \\
\hline $\mathbf{C}^{* *}$ & 0.17 & 3.12 & 1.53 & 2.01 & 1 \\
\hline $\mathbf{D}^{* *}$ & 2.15 & 1.98 & 0.06 & 2.72 & 2 \\
\hline $\mathbf{E}^{* *}$ & 9.61 & 0.10 & 139 & 3.79 & 4 \\
\hline
\end{tabular}

(*) F significativa al 0.01 de margen de error.

$\mathrm{A}^{* *}:$ Se olvida a los Comités de Defensa

$\mathrm{B}^{* *}:$ Se olvida que ya ha pasado antes.

$\mathrm{C}^{* *}$ : Cuando se ocupan zonas de alto riesgo.

$\mathrm{D}^{* *}:$ Cuando se posterga la loma de precauciones.

$\mathrm{E}^{* *}$ : Cuando se espera que otros nos den seguridad.

7. Lo repentino de los desastres hace pensar: Se encuentra que las mujeres significativamente atribuyen los fenómenos naturales a pruebas que Dios nos pone, en tanto los varones consideran que ocurre por acción de la naturaleza. La muestra en general atribuye los desastres a la acción de la naturaleza, seguido por la consideración que son pruebas que Dios nos pone, y no se le atribuye a factores de mala suerte

\section{Cuadro Nro 7}

Resumen de Análisis de Varianza (Razones F) en función a la edad, género y estado civil y medias aritméticas de la muestra total (776 sujeto) respecto a la percepción que los desastres son tan repentinos que pareciera que son

\begin{tabular}{|c|c|c|c|c|c|}
\hline Percepc. & $\begin{array}{c}\text { Edad } \\
\text { F }\end{array}$ & $\begin{array}{c}\text { Gén. } \\
\text { F }\end{array}$ & $\begin{array}{c}\text { E.c. } \\
\text { F }\end{array}$ & $\begin{array}{c}\text { Prom } \\
\text { Gp.T. }\end{array}$ & Rango \\
\hline A $^{* *}$ & 0.97 & $021^{*}$ & 136 & 2.07 & 2 \\
\hline B $^{* *}$ & 019 & 1.41 & 0.19 & 2.51 & 3 \\
\hline C $^{* *}$ & 0.54 & $7.51 *$ & 2.73 & 1.42 & 1 \\
\hline
\end{tabular}

(*) F significativa al 0.01 de margen de error 


\author{
A**: Pruebas que Dios nos pone \\ $B^{* *}:$ Son productos de mala suerte \\ $C^{* *}$ : Ocurren por acción de la naturaleza
}

8. Ocurrencia de un desastre estando uno cerca a un lugar que posee cosa de mucho valor: No se registra efectos de las variables pero el grupo en general señala que se alejarían lo más rápido posible, pero también aparece la segunda opción coger lo que se puede y en tercer lugar parecido al anterior, pedirían a otros que compartan con él, no se registra interés por aprovecharse directamente de la situación.

\title{
Cuadro Nro 8
}

Resumen de Análisis de varianza (Razones $F$ ) en función a la edad, género y estado civil y medias aritméticas de la muestra total (776 sujetos) respecto a lo que haría si estuviera muy cerca de un lugar donde se guardan cosa de mucho valor y ocurriera un desastre de origen natural.

\begin{tabular}{|c|c|c|c|c|c|}
\hline Reacciones & $\begin{array}{c}\text { Edad } \\
\text { F }\end{array}$ & $\begin{array}{c}\text { Gén. } \\
\text { F }\end{array}$ & $\begin{array}{c}\text { E.c. } \\
\text { F }\end{array}$ & $\begin{array}{l}\text { Prom } \\
\text { Gp.T }\end{array}$ & Rango \\
\hline $\mathbf{A}^{* *}$ & 0.54 & 2.47 & 0.32 & 2.03 & I \\
\hline $\mathbf{B}^{* *}$ & 0.54 & 1.09 & 0.02 & 2.86 & 3 \\
\hline $\mathbf{C}^{* *}$ & 0.52 & 011 & 0.58 & 2.55 & 2 \\
\hline $\mathbf{D}^{* *}$ & 1.36 & 1.81 & 0.51 & 3.44 & 3 \\
\hline $\mathbf{E}^{* *}$ & 0.13 & 6.54 & 1.53 & 4.07 & 4 \\
\hline
\end{tabular}

$A * *:$ Me alejo rápidamente

$B^{* *}$ : Vigilo el lugar

$C^{* *}$ : Cojo lo que puedo.

$D^{* *}:$ Pido a otros que compartan conmigo

$E^{* *}$ : Si no aprovecho otros lo harán.

9. Percepciones hacia los simulacros de desastre: La edad y el género interactúan siendo las mujeres de 40 a más años quienes opinan que son una pérdida de tiempo, de la misma forma interactúan la edad con el estado civil en dirección al grupo de separados y divorciados mayores de 40 años. La muestra en general opinan en primer lugar que son útiles para la prevención, aun cuando en segundo lugar opinan que la población los ignora, seguido por la opinión que resultan inútiles porque la realidad es diferente siendo irrelevantes las opiniones que se organizan para justificar sueldo y que es una pérdida de tiempo. (Ver Cuadro Nro. 9). 


\section{Cuadro Nro 9}

Resumen de Análisis de varianza (Razones F) en función a la edad, género y estado civil y medias aritméticas de la muestra total (776 sujetos) respecto a lo que se piensa de los simulacros de prevención de desastres de origen natural

\begin{tabular}{|c|c|c|c|c|c|}
\hline Reacciones & $\begin{array}{c}\text { Edad } \\
\mathbf{F}\end{array}$ & $\begin{array}{c}\text { Gen. } \\
\mathbf{F}\end{array}$ & $\begin{array}{c}\text { E.C. } \\
\text { F }\end{array}$ & $\begin{array}{c}\text { Prom } \\
\text { Gp.T. }\end{array}$ & Rango \\
\hline $\mathbf{A}^{* *}$ & $15.96^{*}$ & 1.95 & 0.6 & 3.98 & 5 \\
\hline $\mathbf{B}^{* *}$ & 0.04 & 1.75 & 3.54 & 3.61 & 4 \\
\hline $\mathbf{C}^{* *}$ & 1.94 & 0.48 & 0.59 & 2.29 & 2 \\
\hline $\mathbf{D}^{* *}$ & 4.01 & 036 & 0.07 & 1.76 & 1 \\
\hline $\mathbf{E}^{* *}$ & 0.09 & 1.84 & 1.21 & 3.30 & 3 \\
\hline
\end{tabular}

(*) F significativa al 0.01 de margen de error

$A^{* *}$ : Es pérdida de tiempo

$B^{* *}$ : Es para justificar los sueldos que ganan.

$C^{* *}$ : La población los ignora.

$D^{* *}:$ Son ensayos para estar prevenidos. $E^{* *}$ : Son inútiles, la realidad es diferente.

10. Percepciones acerca de la ayuda que llega del extranjero: Sólo la edad plantea diferencias para considerar que es ineficaz debido a que la prensa no informa adecuadamente en dirección a las mujeres de cuarenta a más años. Analizando el orden de sus opiniones la muestra estudiada opina que la ineficacia se debe a que se reparte poco, quedándose con el resto, en segundo lugar consideran que es insuficiente.( ver Cuadro Nro 10).

\section{Cuadro Nro 10}

Resumen de Análisis de varianza (Razones f) en función a la edad, género y estado civil y medias aritméticas de la muestra total (776 sujetos) respecto a lo que se piensa del porque no es eficaz la ayuda que llega del extranjero cuando ocurren desastres de origen natural

\begin{tabular}{|c|c|c|c|c|c|}
\hline Pensam. & $\begin{array}{c}\text { Edad } \\
\text { F }\end{array}$ & $\begin{array}{c}\text { Gen } \\
\text { F }\end{array}$ & $\begin{array}{c}\text { E.C } \\
\text { F }\end{array}$ & $\begin{array}{c}\text { Prom } \\
\text { Gp.T }\end{array}$ & Rango \\
\hline $\mathbf{A}^{* *}$ & 8.04 & 0.06 & 131 & 3.46 & 4 \\
\hline $\mathbf{B}^{* *}$ & 0.47 & 0.66 & 031 & 2.69 & 3 \\
\hline $\mathbf{C}^{* *}$ & 3.48 & 5.47 & 0.00 & 2.63 & 2 \\
\hline $\mathbf{D}^{* *}$ & 0.02 & 2.53 & 0.99 & 2.52 & 1 \\
\hline $\mathbf{E}^{* *}$ & 1.10 & 0.16 & 4.84 & 3.65 & 5 \\
\hline
\end{tabular}

(*) F significativa al 0.01 de margen de error.

$A^{* *}:$ La prensa no informa bien

$B^{* *}$ : Todo se queda en los bolsillos de algunos

$C^{* *}$ : Siempre es insuficiente

$D^{* *}:$ Se distribuye poco el resto se la quedan

$E^{* *}$ : Solo favorece a los más necesitados. 
11. La ayuda sería mejor: Opina la muestra en general si se contara con organizaciones de barrio y si el Gobierno diera más ayuda económica. Así mismo se encuentra interacción entre el estado civil con la edad siendo los casados de 24 a menor edad quienes opinan que los desastres son una buena ocasión para hacer negocio. (ver Cuadro Nro. 11).

\section{Cuadro Nro 11}

Resumen de Análisis de varianza (Razones $F$ ) en función a la edad, género y estado civil y medias aritméticas de la muestra total (776 sujetos) respecto a lo que se piensa de como se ayudaría mejor en caso de ocurrir un desastre de origen natural

\begin{tabular}{|c|c|c|c|c|c|}
\hline Pensam. & $\begin{array}{c}\text { Edad } \\
\mathbf{F}\end{array}$ & $\begin{array}{c}\text { Gén. } \\
\mathbf{F}\end{array}$ & $\begin{array}{c}\text { E.C } \\
\mathbf{F}\end{array}$ & $\begin{array}{c}\text { Prom } \\
\text { Gp.T }\end{array}$ & Rango \\
\hline $\mathbf{A}^{* *}$ & 0.95 & 1.64 & 0.02 & 1.70 & 1 \\
\hline $\mathbf{B}^{* *}$ & 2.51 & 0.08 & 3014 & 3.05 & 3 \\
\hline $\mathbf{C}^{* *}$ & 0.58 & 1.75 & 1.74 & 3.14 & 4 \\
\hline $\mathbf{D}^{* *}$ & 2.45 & 128 & 0.00 & 2.43 & 2 \\
\hline $\mathbf{E}^{* *}$ & 0.53 & 2.02 & 0.14 & 4.71 & 5 \\
\hline
\end{tabular}

(*) F significativa al 0.01 de margen de error.

$\mathrm{A}^{* *}$ : Si hay organizaciones de barrio.

$\mathrm{B}^{* *}$ : Si la Cruz Roja invierte más dinero.

$\mathrm{C}^{* *}:$ Los Bomberos fueran más eficientes

$\mathrm{D}^{\star *}:$ El Gobierno diera más ayuda económica.

$\mathrm{E}^{* *}:$ Si se aprovecha para hacer negocio.

B. Las disposiciones psicológicas ante los focos de emergencia de origen social en función al género, edad, estado civil y tipo de ocupación.

1. Desastre de origen social que genera mayor aversión: La edad con el estado civil interactúan respecto a los atentados terroristas como el desastre que genera mayor aversión, en dirección a los solteros entre los 25 a 39 años de edad. En la muestra general estar cerca a los atentados terroristas es lo que genera mayor aversión seguido por estar cerca a una pandilla juvenil y de un asalto a mano armada, y casi no generan aversiones estar cerca de un accidente de tránsito y menos aún estar dentro de un tumulto en un espectáculo público. (Ver Cuadro Nro 12) 


\section{Cuadro Nro 12}

Resumen de Análisis de varianza (Razones $F$ ) en función a la edad, género y estado civil y medias aritméticas de la muestra total (776 sujetos) respecto a la situación ante un desastre de origen social ante el cual JAMAS quisiera encontrarse

\begin{tabular}{|c|c|c|c|c|c|}
\hline $\begin{array}{l}\text { Deseo no } \\
\text { encontrarme }\end{array}$ & Edad F & Gén. F & E.c. F & $\begin{array}{c}\text { Prom } \\
\text { Gp.T. }\end{array}$ & Rango \\
\hline $\mathbf{A}^{* *}$ & 4.19 & 1.92 & 2.68 & 2.86 & 2 \\
\hline $\mathbf{B}^{* *}$ & 3.43 & 0.48 & 0.69 & 3.91 & 4 \\
\hline $\mathbf{C}^{* *}$ & 1.56 & 1.02 & 0.16 & 2.01 & 1 \\
\hline $\mathbf{D}^{* *}$ & 4.33 & 0.00 & 2.81 & 2.86 & 21 \\
\hline $\mathbf{E}^{* *}$ & 0.31 & 0.04 & 1.18 & 3.35 & 3 \\
\hline
\end{tabular}

$A^{* *}:$ Frente a una pandilla juvenil.

$B^{* *}:$ Dentro de un tumulto dentro de un espectáculo.

$C^{* *}$ : Cerca de un atentado terrorista.

$D^{* *}$ : En un asalto a mano armada.

$E^{* *}:$ Ante un accidente de tránsito.

2. Reacción emocional ante desastres de origen social: El género influye sobre las reacciones de ansiedad y miedo en dirección a las mujeres y de igual forma para sacar provecho de la situación. La muestra en general comunican que su reacción será «corre hasta llegar a un lugar seguro», y dar la voz de alarma; en tercer lugar está la reacción de miedo y ansiedad, en cambio es irrelevante, quedarse en el lugar para ver el final y sacar provecho de la situación ( Ver Cuadro Nro.13).

\section{Cuadro Nro 13}

Resumen de Análisis de varianza (Razones F) en función a la edad, género y estado civil y medias aritméticas de la muestra total (776 sujetos) respecto a lo que liaría en caso de encontrase inevitablemente ante un desastre de origen social.

\begin{tabular}{|c|c|c|c|c|c|}
\hline $\begin{array}{c}\text { Lo que se } \\
\text { haría }\end{array}$ & $\begin{array}{c}\text { Edad } \\
\mathbf{F}\end{array}$ & $\begin{array}{c}\text { Gén. } \\
\mathbf{F}\end{array}$ & $\begin{array}{c}\text { E.C } \\
\mathbf{F}\end{array}$ & $\begin{array}{c}\text { Prom } \\
\text { Gp.T }\end{array}$ & Rango \\
\hline $\mathbf{A}^{* *}$ & .048 & 4.82 & 1.00 & 1.62 & 1 \\
\hline $\mathbf{B}^{* *}$ & 038 & 0.38 & 0.00 & 1.99 & 2 \\
\hline $\mathbf{C}^{* *}$ & 0.84 & $19.15 *$ & 0.53 & 3.09 & 3 \\
\hline $\mathbf{D}^{* *}$ & 239 & $6.84 *$ & 4.33 & 3.73 & 4 \\
\hline $\mathbf{E}^{* *}$ & 1.11 & $18.02 *$ & 0.00 & 4.56 & 5 \\
\hline
\end{tabular}

(*) F significativa al 0.01 de margen de error.

A**: Corre hasta llegar a un lugar seguro.

$B^{* *}$ : Dar la voz de alarma

$C^{* *}$ : Sufrir de un ataque de ansiedad. 
$D^{* *}:$ Ale quedo para ver en que termina.

$E^{* *}$ : Si se puedo saco provecho de la situación.

3. Reacciones en caso de enterarse de un acto de sabotaje: Darían avisa a la policía, los varones entre los 25 a 39 años, encontrándose además interacción entre el género y el estado civil para la misma reacción y para hacer que otros den la voz de alarma. En general la muestra comunica que vería la forma de avisar a la policía en segundo lugar se alejarían de la situación, en tercer lugar contarían a terceros para que sean éstos los que den la alarma, pero no guardarían silencio ni el temor los haría callar. (Ver Cuadro Nro. 14).

\section{Cuadro Nro 14}

Resumen de Análisis de varianza (Razones F) en función a la edad, género y estado civil y medias aritméticas de la muestra total (776 sujetos) respecto a lo que haría en caso de enterarse de una acción de sabotaje

\begin{tabular}{|c|c|c|c|c|c|}
\hline $\begin{array}{c}\text { Lo que se } \\
\text { haría }\end{array}$ & $\begin{array}{c}\text { Edad } \\
\mathbf{F}\end{array}$ & $\begin{array}{c}\text { Gen. } \\
\mathbf{F}\end{array}$ & $\begin{array}{c}\text { E.C. } \\
\mathbf{F}\end{array}$ & $\begin{array}{c}\text { Prom } \\
\text { Gp.T. }\end{array}$ & Rango \\
\hline $\mathbf{A}^{* *}$ & 3.44 & 0.02 & 0.51 & 3.49 & 54 \\
\hline $\mathbf{B}^{* *}$ & 50.45 & 15.68 & 0.18 & 3.57 & 5 \\
\hline $\mathbf{C}^{* *}$ & 2.04 & 3.42 & 2.61 & 2.63 & 2 \\
\hline $\mathbf{D}^{* *}$ & 5.69 & $8.88 *$ & 0.04 & 230 & 1 \\
\hline $\mathbf{E}^{* *}$ & 0.50 & 429 & 1.64 & 3.00 & 3 \\
\hline
\end{tabular}

(*) F significativa al 0.01 de margen de error.

$\boldsymbol{A} * *:$ Guardar silencio por temor a represalias.

$\boldsymbol{B}^{* * *}$ : El temor me haría callar

$C^{* *}$ : Me alejo del lugar lo más pronto posible.

$D^{* *}$ : Comunico a la Policía.

$\boldsymbol{E}^{* *}$ : Cuento a otro para que de la alarma.

4. Percepción de lo que se haría para evitar la proliferación de pandillas juveniles: Las mujeres consideran que el trato afectuoso a los jóvenes es una buena medida, por otro lado los sujetos de 24 a 39 años consideran que es muy efectivo evitar para los suyos la influencia de las malas juntas en tanto que las mujeres de 40 años consideran que es mejor alejarse de los lugares de riesgo, mientras que los jóvenes de 15 a 24 años opinan que la mejor forma es colaborando con las organizaciones juveniles. Este último punto de vista ocupa el primer lugar en la muestra general, seguido por el trato afectuoso a los jóvenes y consideran que no arregla nada alejándose o denunciando a la policía.(Ver Cuadro Nro. 15) 


\section{Cuadro Nro 15}

Resumen de Análisis de varianza (RazonesF) en función a la edad, género y estado civil y medias aritméticas de la muestra total (776 sujetos) respecto a lo que haría para evitar la proliferación de pandillas juveniles

\begin{tabular}{|c|c|c|c|c|c|}
\hline $\begin{array}{c}\text { Lo que se } \\
\text { haría }\end{array}$ & $\begin{array}{c}\text { Edad } \\
\mathbf{F}\end{array}$ & $\begin{array}{c}\text { Gen. } \\
\mathbf{F}\end{array}$ & $\begin{array}{c}\text { E.c. } \\
\mathbf{F}\end{array}$ & $\begin{array}{c}\text { Prom } \\
\text { Gp.T. }\end{array}$ & Rango \\
\hline $\mathbf{A}^{* *}$ & 0.00 & 0.19 & 0.18 & 3.43 & 5 \\
\hline $\mathbf{B}^{* *}$ & 0.77 & $0.23 *$ & 0.18 & 2.91 & 2 \\
\hline $\mathbf{C}^{* *}$ & $5.50 *$ & 3.92 & 1.22 & 2.76 & 3 \\
\hline $\mathbf{D}^{* *}$ & 3.53 & 2.18 & 0.41 & 338 & 4 \\
\hline $\mathbf{E}^{* *}$ & 7.74 & 0.01 & 0.04 & 2.52 & 1 \\
\hline
\end{tabular}

${ }^{\star}{ }^{*}$ F significativa al 0.01 de margen de error.

A**: Denunciarlos a la policía.

$B^{* *}:$ Tratar afectuosamente a los jóvenes.

$C^{* *}:$ Evitaría para los míos las malas juntas

$D^{* *}:$ Alejarme de los lugares de riesgo.

$E^{* *}$ : Colaborar con organizaciones juveniles.

5. Percepción de lo que se haría para evitar pérdidas de vidas humanas por tumultos en los espectáculos públicos: Los mayores de 40 años opinan que las municipalidades no deben dar licencia en tanto que los sujetos de 24 o menos edad consideran que es inevitable. La muestra en general asume que se evitaría educando a la gente para precaver, en segundo lugar si hubiera mayor custodia policial.(Ver Cuadro Nro. 16)

\section{Cuadro Nro 16}

Resumen de Análisis de varianza (Razones F) en función a la edad, género y estado civil y medias aritméticas de la muestra total (776 sujetos) respecto a lo que haría para evitar la pérdida de vidas por tumultos en espectáculos públicos

\begin{tabular}{|l|c|c|c|c|c|}
\hline Lo que se haría & $\begin{array}{c}\text { Edad } \\
\text { F }\end{array}$ & Gén. F & E.C F & $\begin{array}{c}\text { Prom } \\
\text { Gp.T. }\end{array}$ & Rango \\
\hline $\mathrm{A}^{* *}$ & $20.63 *$ & 034 & 0.45 & 3.48 & 4 \\
\hline $\mathrm{B}^{* *}$ & 2.71 & 022 & 0.03 & 2.54 & 2 \\
\hline $\mathrm{C}^{* *}$ & 291 & 0.80 & 2.24 & 2.13 & 1 \\
\hline $\mathrm{D}^{* *}$ & $4.83^{*}$ & 1.58 & 0.64 & 3.80 & 5 \\
\hline $\mathrm{E}^{* *}$ & 1.47 & 0.95 & 2.04 & 3.01 & 3 \\
\hline
\end{tabular}

(*) F significativa al 0.01 de margen de error.

A** : La Municipalidad no debe dar licencias.

$\mathrm{B}^{* *}$ : Dar mayor custodia policial.

$\mathrm{C}^{* *}$ : Educando a las personas para preveer

$\mathrm{D}^{* *}$ : Es inevitable la gente no aprende

$\mathrm{E}^{* *}$ : Sancionar drásticamente a los organizadores. 
6. Reacción en caso recibiera una invitación para incorporarse a una brigada de defensa civil: La muestra considera que finalmente ocurrió lo que deseaban, en segundo lugar consideran que guardarían su distancia por tener asuntos más importantes, pero no se considera como una pérdida de tiempo.(Ver Cuadro No. 17).

\section{Cuadro Nro 17}

Resumen de Análisis de varianza (Razones F) en función a la edad, género y estado civil y medias aritméticas de la muestra total (776 sujetos) respecto a lo que pensaría si fuera convocado para participar en una brigada de defensa civil

\begin{tabular}{|c|c|c|r|r|c|}
\hline $\begin{array}{c}\text { Lo que se } \\
\text { haría }\end{array}$ & $\begin{array}{c}\text { Edad } \\
\text { F }\end{array}$ & $\begin{array}{c}\text { Gen. } \\
\text { F }\end{array}$ & $\begin{array}{c}\text { E.C } \\
\text { F }\end{array}$ & $\begin{array}{c}\text { Prom } \\
\text { Gp.T. }\end{array}$ & Rango \\
\hline $\mathbf{A}^{* *}$ & 1.87 & 0.43 & 1.51 & 1.99 & 1 \\
\hline $\mathbf{B}^{* *}$ & 1.86 & 2.14 & 0.01 & 2.34 & 3 \\
\hline $\mathbf{C}^{* *}$ & 1.39 & 1.12 & 2.62 & 2.27 & 2 \\
\hline $\mathbf{D}^{* *}$ & 0.21 & 0.03 & 0.92 & 3.39 & 4 \\
\hline
\end{tabular}

(*) $F$ significativa al 0.01 de margen de error.

A**: Que por fin ocurrió lo que deseaba.

$B^{* *}$ : Que tiene asuntos más importantes.

$C^{* *}$ : Que mejor guarda su distancia.

$D^{* *}:$ Que siempre es una perdida de tiempo.

7. Creencia de intereses ocultos que evitan que los desórdenes sociales terminen: Los examinados consideran que la prensa sensacionalista mantiene los desórdenes sociales, en segundo lugar los partidos políticos y en tercer lugar los grupos económicos no se consideran que sean hechos insolubles y menos aún porque la gente actúa con generosidad. (Ver Cuadro Nro.18).

\section{Cuadro Nro 18}

Resumen de Análisis de varianza (Razones $F$ ) en función a la edad, género y estado civil y medias aritméticas de la muestra total (776 sujetos) respecto a lo que cree si hay quienes sacan provecho de los desórdenes sociales y es por ello que no terminan

\begin{tabular}{|c|c|c|c|c|c|}
\hline $\begin{array}{c}\text { ¿Sacan } \\
\text { provecho? }\end{array}$ & $\begin{array}{c}\text { Edad } \\
\text { F }\end{array}$ & $\begin{array}{c}\text { Gen. } \\
\text { F }\end{array}$ & $\begin{array}{c}\text { E.c. } \\
\text { F }\end{array}$ & $\begin{array}{c}\text { Prorn } \\
\text { Gp.T. }\end{array}$ & Rango \\
\hline $\mathbf{A}^{* *}$ & 0.21 & 0.03 & 0.92 & 1.93 & 1 \\
\hline $\mathbf{B}^{* *}$ & 0.85 & 0.92 & 1.04 & 2.18 & 2 \\
\hline $\mathbf{C}^{* *}$ & 0.69 & 3.82 & 2.84 & 2.72 & 3 \\
\hline $\mathbf{D}^{* *}$ & 0.72 & 093 & 2.03 & 3.68 & 4 \\
\hline $\mathbf{E}^{* *}$ & 1.28 & 0.01 & 0.44 & 4.98 & 5 \\
\hline
\end{tabular}

(*) F significativa al 0.01 de margen de error.

$A^{* *}:$ Sí, la prensa sensacionalista.

$B^{* *}:$ Sí, los partidos políticos.

$C^{* *}:$ Sí, Los grupos económicos. 
$D^{* *}:$ No, son hechos que tienen solución

$E^{* *}:$ No, la gente actúa con generosidad

\section{DISCUSIÓN}

Las Disposiciones Psicológicas entendidas como proceso psicológico de redes de significados que predisponen al individuo para pensar, sentir y actuar de una forma determinada entre varias posibles, que en la presente investigación se manifiestan como priorizaciones de conductas ante los focos de emergencia de origen natural y social no recibe la influencia del género, edad, estado civil y tipo de ocupación ya que de 87 análisis de varianza efectuados en función a cada una de estas variables respecto a cada una de las Disposiciones Psicológicas, la edad influye sólo en el 16\% lo que arroja un Ji cuadrado en favor a efectos del azar igual a 35, para el género el $18 \%$ con un valor $\mathrm{Ji}$ cuadrado de 29 y para estado civil el $3 \%$ con un Ji cuadrado de 75, el tipo de ocupación no llega ni al $1 \%$ y las interacciones de edad con el genero, con estado civil, tipo de ocupación, y el género con el estado civil y el tipo de ocupación son irrelevantes, de forma tal que podemos afirmar que las Disposiciones Psicológicas hacia los focos de desastre de origen natural y social no son influenciadas por el género, edad, tipo de ocupación y estado civil, debido a que por las características aversivas de estos fenómenos, las reacciones humanas suelen ser comunes, con variaciones en intensidades y estilos pero no diferentes, ya que del tipo de reacción que den las personas depende en gran medida conservar su vida o su integridad física, en conclusión los estudios al respecto deben enfatizar en la jerarquización de respuestas de la población en general sin tener en cuenta que alguna de las variables estudiadas la afecten significativamente.

\section{CONCLUSIONES}

1. Se acepta la hipótesis nula por la que se afirma que las variables: edad, género, estado civil y el tipo de ocupación no influyen sobre las Disposiciones Psicológicas hacia los estados de emergencia de origen natural y social.

2. Se acepta la hipótesis nula por la que se afirma que las variables: edad, género, estado civil y el tipo de ocupación no interactúan entre si influyendo sobre las disposiciones psicológicas hacia los estados de emergencia de origen natural y social.

3. Ante los desastres de origen natural, los que producen mayor miedo son: terremotos, incendios y los tsunamis no así los huaycos, huracanes y tormentas.

En caso que ocurriera un desastre quisieran que suceda cuando están en sus hogares. Al finalizar el desastre natural lo primero que harían si estuvieran lejos de su hogar es regresar a cualquier costo, luego pedirían ayuda y algunos irían al foco del desastre por otro lado pocos rezarían o pensarían que hacer.

Terminado el desastre quedarían con temor a que se repita y arrepentidos por no haber tomado precauciones, muy pocos se sentirían amargados y resentidos con todo. Las instituciones que son percibidas como efectivas ante los desastres son Defensa Civil, la Cruz Roja y los Bomberos, no así el Gobierno y menos aún las 
Municipalidades.

Consideran que los efectos de los desastres son mayores porque la gente ocupa zonas de alto riesgo, porque se postergan las medidas preventivas y porque se olvidan de formar comités de defensa y que ya pasó antes y por ende que en cualquier momento se repetirá.

Por otro lado consideran que la ocurrencia de los desastres naturales se debe a la acción de la naturaleza y también a pruebas que Dios nos manda, en cambio no se le atribuye a la mala suerte.

Se encuentra tendencias marcadas al oportunismo para quedarse con objetos de valor material si las circunstancias lo permiten.

Por otro lado, los simulacros son percibidos como útiles como medida preventiva sin embargo consideran que suelen ser ignorados por uno y por lo demás.

La percepción que se tiene de la ayuda extranjera es que se reparte sólo una parte de ella, y que alguien se queda con el resto. La ayuda sería más efectiva si se contara con organizaciones de barrio y si habría más ayuda económica.

4. Ante los desastres de origen social, los que generan alta ansiedad y miedo son los atentados terroristas, seguido por las pandillas juveniles y los asaltos a mano armada, no así los accidentes de tránsito ni los tumultos en los espectáculos públicos. La primera reacción de las personas es la de correr hasta llegar a un lugar seguro y dar la voz de alarma, no hay disposición para quedarse a ver el final. Ante un sabotaje se avisa a la policía y no se quedarían callados. Puede evitarse que proliferen las pandillas juveniles, si colaboramos con organizaciones juveniles y si damos afecto a los jóvenes, agrava las cosas si solo nos alejamos y denunciamos a la policía. Para evitar la pérdida de vidas por los tumultos en espectáculos públicos se debe educar a la gente y dar mayor custodia policial.

Si se fuera invitado a pertenecer a una brigada de defensa civil se aceptaría con gusto, no se interpreta como una pérdida de tiempo.

Finalmente, consideran que los desastres sociales perduran porque hay intereses ocultos en la prensa sensacionalista, los partidos políticos y en los grupos de poder económico, pero que a pesar de todo son problemas que pueden resolverse.

\section{BIBLIOGRAFÍA}

Brown, Roger. Psicología Social. Edit. Siglo XXI. segunda edición en español 1979.

Khol David. Psicología de las Organizaciones, Problemas Contemporáneos. Prentice Hall 1993.

Kuch Joanne. Aprendiendo a conocerme. Ed.Globe Boock Company. 1994

Gerald, G. Davidson y J.Nweale. Psicología de la Conducta anormal. Limusa 1991. 